\section{Elcio Juliato Piovesan \\ Helio Ghizoni Teive \\ Luciano de Paola \\ Carlos Eduardo Silvado \\ Ana Crippa \\ Vanise Campos Gomes Amaral \\ Marcos Vinicius Della Colleta \\ Fabrizio Di Stani \\ Lineu Cesar Werneck}

\title{
Uncontrolled headache induced \\ by oxcarbazepine
}

Received: 2 August 2007

Accepted in revised form: 20 September 2007

Published online: 25 October 2007

E.J. Piovesan $(\varangle) \bullet$ H.G. Teive $\bullet$ L. de Paola • C.E. Silvado • A. Crippa • V.C.G. Amaral • M.V. Della Colleta • L.C. Werneck Headache Clinic, Neurology Service, Department of Internal Medicine,

Hospital de Clínicas,

Federal University of Paraná,

Rua General Carneiro 181,

120 andar, sala 1236 ,

CEP-80060-900, Curitiba - Paraná, Brazil

e-mail: piovesan@avalon.sul.com.br

Tel.: +41-3283-5512

Fax: +41-3283-5512

F. Di Stani

Department of Neurological Sciences -

University of Rome "La Sapienza"

Rome, Italy

\begin{abstract}
Headache induced by acute exposure to a specific drug constitutes an idiosyncratic side effect. Metabolic imbalance appears as the leading aetiology, among several other hypotheses. Either primary headaches show a higher susceptibility to this idiosyncratic reaction or a druginduced primary headache evolves in intensity and duration, becoming uncontrolled until the complete discontinuation of the drug in consideration. The goal of this study is to describe three patients diagnosed with migraine and epilepsy (both under control) who evolved into status migrainosus after the introduction of oxcarbazepine (OXC), as part of a switch off from carbamazepine (CBZ). Twenty-four to seventy-two hours following the switch, all patients
\end{abstract}

developed intractable headache, despite the use of different symptomatic drugs. Complete recovery of the headache symptoms occurred only after OXC was discontinued. We discuss the potential mechanisms associated to $\mathrm{OXC}$ and status migrainosus, druginduced headaches and uncontrolled headaches.

\section{Introduction}

Headaches induced by acute substance use or exposure are common conditions in which there is a clear relationship between the onset of symptoms and the intake of one drug for therapeutic purposes. The Second Edition of the International Classification of Headache Disorders (ICHD-II) subdivided this group in 11 subgroups [1]. Nitric oxide, phosphodiesterase inhibitors, carbon monox- ide, alcohol, monosodium glutamate, cocaine, cannabis, histamine and calcitonin gene-related peptide are the main drugs that induce headaches. However, other drugs or therapeutic agents may be involved, depending on various patient susceptibility factors.

Migraineurs are physiologically and perhaps psychologically hyper-responsive to a variety of internal and external stimuli. Alcohol, food and food additives, chemical, drug ingestion and withdrawal have all been reported 
to provoke or activate migraine in susceptible individuals1. The association is often based on anecdotal data and reports of adverse drug reactions. Thus, the mere use of a given drug leading to headache does not prove causation, because these common events happen commonly and probably reflect a pure coincidence. Headache can be a symptom of a systemic disease, and drugs given to treat such a condition will be associated with headache. In acute migraine drug trials, headache, as well as associated symptoms, is listed as an adverse drug reaction despite the fact that it is a symptom of the treated disorder and not the result of the treatment. However some acute or chronic substance exposures have been proven to be causally related to headache. Medications are a well known cause of secondary headache, especially in the elderly.

A variety of drugs can induce headache (Table 1). Interestingly, some are commonly employed as either preventive or symptomatic medications in the treatment of migraine or other headaches [2,3]. Vasodilation and increase of intracranial pressure are some of the putative mechanisms underlying drug-related headaches.

Three patients in whom oxcarbazepine (OXC) is potentially implicated in the development of status migrainosus are presented. We reviewed the literature and discuss concepts that may be related to such cases in the context of uncontrolled headache and drug-induced headaches.

\section{Materials and methods}

Patient 1

A 33-year-old female was referred to the Headache Clinic due to severe and intractable headache. She started having headaches at the age of 10, with unilateral, pulsating headache, associated with photophobia, phonophobia, nausea and vomiting, lasting from 6 to $18 \mathrm{~h}$. Typically, the headache attacks were related to the menses and tended to subside entirely following the use of $50 \mathrm{mg}$ of sumatriptan. At age 16 the patient presented with secondarily generalised seizures. MR imaging showed a small mass on the left temporal lobe, consistent with a dysembryoplastic neuroepithelial tumour. She was put on carbamazepine (CBZ) and subsequently on levetiracetam with poor results. CBZ (at daily dose of $1400 \mathrm{mg}$ ) was switched to OXC (at daily dose of $2100 \mathrm{mg}$ ), as an attempt to improve her seizure control. On day 2 of OXC a diffuse pulsating headache of severe intensity, associated with nausea and vomiting and aggravated by physical activity, was reported. By the time of her evaluation at the outpatient clinic the headache had proven to be resistant to oral sumatriptan (100 mg), IV metroclopramide, Cox-2 inhibitors, IM nonsteroid anti-inflammatory (NSAI), IV dexamethasone (4 $\mathrm{mg}$ ) and IV chlorpromazine. She was admitted for fluid hydration. OXC was discontinued and CBZ was reintroduced leading to complete headache cessation within a 48 -h period. Sodium levels were not requested during admission.

\section{Patient 2}

A 38-year-old female was referred to the Headache Clinic due to disabling headaches. At age 13 she started with visual and/or sensory symptoms evolving gradually over approximately one hour, followed by headache. The latter was unilateral, pulsating and moderate to severe in intensity pain, lasting up to $24 \mathrm{~h}$. Associated symptoms included nausea and vomiting. At the onset of symptoms the attacks occurred 3 times a month but frequency had increased significantly over the past 8 years. MR imaging showed a large vascular malformation in the right occipital lobe. An attempt at intravas-

Table 1 Drugs that may induce headache or worsen pre-existing headache [1-3]

\begin{tabular}{llll}
\hline Acetazolamine & Didanosine & Isoniazid & Perhexiline \\
Ajmaline & Dihydralazine & Meprobamate & Primidone \\
Amantadine & Dihydroergotamine & Methaqualone & Propranolol \\
Antihistaminics & Dipyridamole & Metronidazole & Prostacyclines \\
Atenolol & Disopyramide & Metoprolol & Ranitidine \\
Barbiturates & Disulfiram & Morphine & Rifampicin \\
Beta-interferon & Ergotamine & Nalidixic acid & Sildenafil \\
Bromocriptine & Ethynilestradiol & Nifedipine & Tamoxifen \\
Caffeine & Gestagens & Nitrofurantoin & Tetracycline \\
Calcium antagonists & Glycerol trinitrate & Nitrates & Theophylline \\
Carbimazol & Glycosides & Non steroidal anti-inflammatory & Thiamazole \\
Chinidine & Griseofulvin & Octreotide & Trimethoprim+sulfamethoxazole \\
Chloroquine & Guanathidine & Oestrogens & Triptans \\
Cimetidine & Immunoglobulins & Omeprazole & Verapamil \\
Clofibrate & Indomethacin & Ondansetron & Vitamin A \\
Codeine & Interferons & Paroxitine & Oxcarbazepine \\
Danazol & Isosorbide dinitrate & Pentoxifylline & \\
\hline
\end{tabular}


cular treatment (embolisation) was complicated by intracerebral haemorrhage (including intraventricular bleeding) that required surgical drainage and the placement of a ventriculoperitoneal shunt. Shortly after the procedures, simple partial seizures with secondary generalisation developed. She was put on CBZ 600 tid and clobazam $10 \mathrm{mg} /$ day. In spite of adequate seizure control the patient complained of dyspepsia and weight gain. CBZ was replaced by OXC $1200 \mathrm{mg}$ (daily dosage). Hours after the switch the headache transformed into daily, severe, bilateral, pulsating pain, accompanied by nausea and vomiting, photo- and phonophobia, increasing with physical effort. The patient was admitted and treated with IM NSAI, IV metroclopramide, IV dexamethasone and IV chlorpromazine with virtually no results. Sodium levels were not obtained. OXC was withdrawn and $\mathrm{CBZ}$ was re-established, leading to complete remission of the symptoms within a 3-day period.

\section{Patient 3}

A 30-year-old female was referred to the Headache Clinic due to intractable epilepsy. Headaches started at age 12, with bilateral, pulsating and severe pain, associated with nausea, vomiting, photoand phonophobia, lasting up to $32 \mathrm{~h}$. Headaches occurred twice a month and were easily treated by oral isometheptene. History was also positive for primary generalised epilepsy. Interestingly, the patient was seizure-free on $1200 \mathrm{mg}$ (daily dose) of CBZ. For unclear reasons the primary physician decided to switch the patient to OXC $1800 \mathrm{mg}$ (daily dose), after which she developed severe, pulsating, holocranial pain, increased by movement and associated with nausea, vomiting, photo- and phonophobia. IM metroclopramide, IV ketoprofen and SC sumatriptan were tried at the outpatient clinic. The patient was admitted and tried on IV dexamethasone and IV chlorpromazine with no results. OXC was discontinued and CBZ reintroduced. The pain subsided over a 3-day period.

All subjects provided written informed consent, as required by the appropriate local committee (Human Ethical committee of Universidade Federal do Paraná) on the protection of research subjects.

\section{Discussion}

We report on three cases of OXC-induced difficult-to-control pain suggesting status migrainosus and subsequently bona fide intractable headache. We felt that an association between both headache and $\mathrm{OXC}$, status migrainosus and $\mathrm{OXC}$ and intractable headache and $\mathrm{OXC}$ could be clearly established, as symptoms developed shortly after the introduction of the drug and completely subsided following its withdrawal.

OXC is a new antiepileptic (AED), available since 1990, which has been proven effective in the treatment of partial seizures [4]. Hyponatraemia, defined as serum sodium concentration of $134 \mathrm{mEq} / \mathrm{l}$ or less, tends to occur in about $30 \%$ of OXC-treated patients and may be severe (i.e., serum sodium below $128 \mathrm{mEq} / \mathrm{l}$ ) in $12 \%$ of the patients [5]. This effect is thought to be mediated by either a direct action of the drug on the renal collecting tubules or an enhancement of their responsiveness to circulating antidiuretic hormone [6].

Serum sodium levels maybe relevant, as shown in a recent paper describing OXC-induced headache [7]. That was a single case in which hyponatraemia $(131 \mathrm{mEq} / \mathrm{l})$ was detected during the course of the headache and improved (141 mEq/l) after OXC withdrawal, when the patient became free of symptoms. Hyponatraemia manifests itself by headache, dizziness and lethargy, though such symptoms are rarely seen with serum sodium levels above 125 $\mathrm{mEq} / 18$. However, hyponatraemia might induce headache in predisposed subjects before the appearance of overt encephalopathy, particularly if associated with other drugs. In the previously described case of OXC-induced headache the patient had a positive family history for headache (probably migraine headache) [7].

OXC was evaluated as preventive therapy in patients suffering from migraines in a multicentre, double-blind, randomised, placebo-controlled, parallel-group study. The double-blind treatment between the OXC and placebo groups was not statistically significant. In this study, headache, as a side effect in migraine patients, was reported in $5.9 \%$ of the cases [9]. Regretfully, clinical features of this specific population were not described in the study.

The IHS defines status migrainosus as an attack of migraine, the headache phase of which last more than $72 \mathrm{~h}$ whether it is treated or not [1]. Factors responsible for triggering status migrainosus include emotional stress, depression, abuse of medications, anxiety, diet, hormonal factors and multiple nonspecific factors [10]. Status migrainosus may be secondary to an acute neurologic disorder. However, acute central nervous system events can trigger an otherwise typical migraine.

Patients with status migrainosus can evolve to intractable headache. Intractable is defined as uncontrollable, refractory or unmanageable [11], and thus the term can be used for a headache that requires either acute or preventive treatment [12]. Recently, criteria of uncontrolled headache have been suggested for preventive treatment: failure of at least four classes, where three should come from 1-4 groups of medicines, including (1),-blockers; (2) anticonvulsants; (3) calcium channel blockers; (4) tricyclic antidepressants; (5) other treatments with at least one positive randomised controlled trial; (6) non-steroidal anti-inflammatory drugs; and (7) metabolic enhancers, such as vitamin B2 or coenzyme Q10.

Although acute intractable headache has not been sug- 
gested until the present moment, we used this term to refer to the three cases that used different lines of the acute treatment for migraine with no success, for a period over $72 \mathrm{~h}$. Only after the OXC was discontinued did the pain subside, reinforcing a direct relationship between OXC and pain in those cases.

When a new headache occurs for the first time in close temporal relation to substance exposure, it is coded as a secondary headache attributed to the substance. This is also true if the headache has the characteristics of migraine, tension-type headache or cluster headache. When a pre-existing, primary headache, as in our cases, is made worse in close temporal relation to substance exposure, there are two possibilities, and careful judgement is required [1]. The patient can either be given only the diagnosis of the pre-existing primary headache or be given both this diagnosis and the diagnosis of headache attributed to the substance. A very close temporal relationship to the substance exposure, a marked worsening of the preexisting headache, very good evidence that the substance can aggravate the primary headache and, finally, improvement or resolution of the headache after termination of effect of the substance [1] are required. Headaches attributed to medication are not very well defined in the literature but most are dull, continuous, diffuse and moderate to severe intensity. The diagnostic criteria are described in Table 2.

We devised two working hypothesis regarding our 3 cases. First, OXC-induced headache and the pain were directly related to acute medication and symptoms were exclusively related to OXC. Secondly, OXC-induced headache and the pain were directly related to acute medication, however, the patients, who had a primary headache history - in these cases, migraine - developed a migraine attack that progressed to status migrainosus, and finally intractable headache (Fig. 1). We will provide substantial evidence for both hypotheses.

Symptoms may be related exclusively to OXC as their onset took place one hour after its introduction and patients experienced complete remission within $72 \mathrm{~h}$ following OXC withdrawal. Standard medications for the treatment of acute migraine attacks failed to resolve the symptoms in all patients. Interestingly enough, headaches induced by medication are generally not severe in intensity and the nausea and vomiting promptly subside. That was not the case in our patients, who required admission, IV fluids and IV metoclopramine.

OXC evolving into status migrainosus is suggested by the presence of severe persistent headache, often associated with nausea and vomiting, seen in all our patients.
It seems that status migrainosus could be induced by OXC as this drug produces hyponatraemia, an atypical situation of the brain. Unfortunately we are unable to provide complete data in that regard, as sodium levels were not monitored during admission in all cases. The literature suggests that in newly diagnosed cases of status migrainosus a secondary aetiology must be sought after and OXC may be the case in our patients. The interactions of OXC and other medicines utilised in our patients could also explain the induced status migrainosus, but the relationship between headache and OXC was seemingly clear.

Table 2 International Headache Society classification for headache as an acute adverse event attributed to medication used for other indications [1]

8.1.10. Diagnostic criteria:

A. Headache fulfilling criteria $\mathrm{C}$ and $\mathrm{D}$

B. Use of a medication for a therapeutic indication other than headache

C. Headache develops within minutes to hours after use

D. Headache resolves within $72 \mathrm{~h}$ after cessation of use

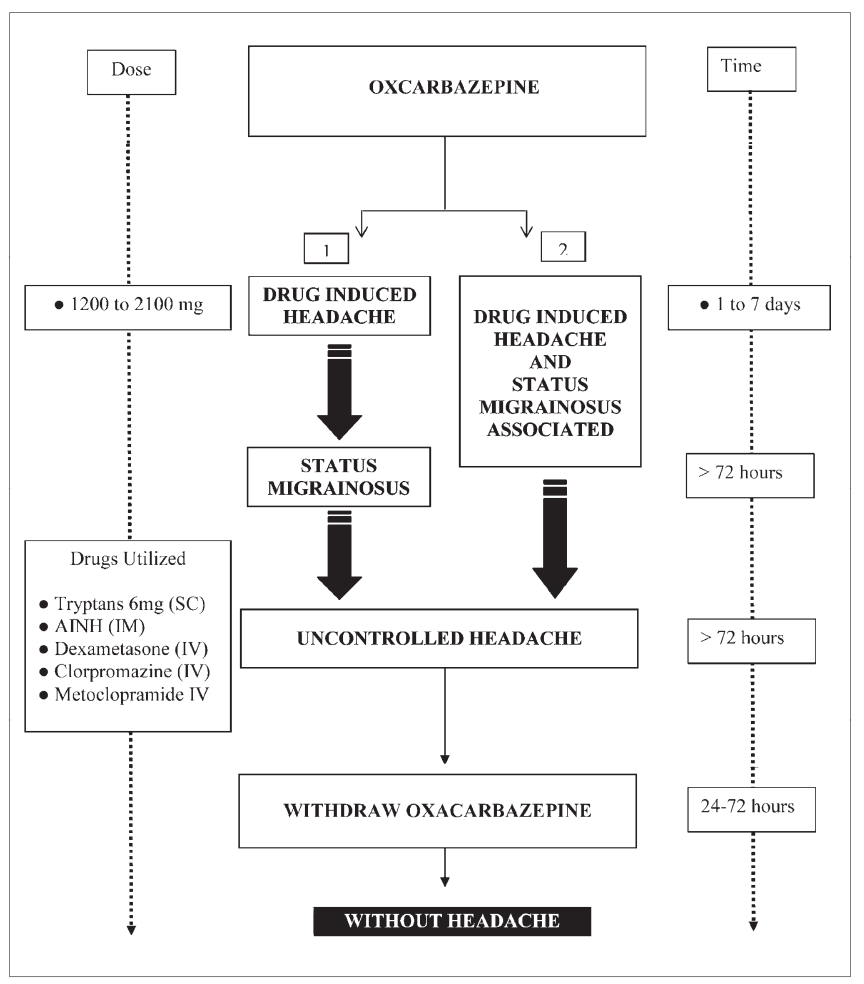

Fig. 1 Sequence of the events of OXC-induced headache and status migrainosus 


\section{References}

1. Headache Classification Committee of the International Headache Society. The International Classification of Headache Disorders, 2nd Edition (2004) Cephalalgia 24 [Suppl 1]:1-160

2. Askmark H, Lundberg PO, Olsson S (1989) Drug related headache. Headache 29:441-444

3. Monteiro JM, Dalhof CG (2000) Single use of substances. In: Olesen J, Tfelt-Hansen P, Welch KMA, (eds) The headaches. Lippincott Williams \& Wilkins, Philadelphia 861-869

4. Glauser TA (2001) Oxcarbazepine in the treatment of epilepsy.

Pharmacotherapy 21:904-919

5. Dong X, Leppik IE, White J, Rarick J (2005) Hyponatremia from oxcarbazepine and carbamazepine. Neurology 65:1976-1978
6. Sachdeo RC, Wasserstein A, Mesenbrink PJ, D’Souza J (2002) Effects of oxcarbazepine on sodium concentration and water handling. Ann Neurol 51:613-620

7. Palmieri A (2007) Oxcarbazepineinduced headache. Cephalalgia 27:91-93

8. Goh KP (2004) Management of hyponatremia. Am Fam Physician 69:2387-2394

9. Saper J, Silberstein S, Somogyi M, McCague K (2005) Efficacy and safety of oxcarbazepine in patients with migraine headaches: a multicenter, double-blind, randomized, placebocontrolled study. Neurology (P05):152

9. Couch JR, Diamond S (1983) Status migrainosus: causative and therapeutic aspects. Headache 23:94-101
10. Oxford English Dictionary. http//dictionary.oed.com. Last accessed 20 March 2007

11. Goadsby PJ, Schoenen J, Ferrari MD et al (2006) Towards a definition of intractable headache for use in clinical practice and trials. Cephalalgia 26:1168-1170

12. Silberstein SD, Lipton RB, Goadsby PJ, Smith RT (1999) Migraine diagnosis and treatment. In: Headache in primary care. Isis Medical Media, Oxford, pp 53-82 